\title{
ARQUEOLOGÍA EN UN AMBIENTE DE CIENCIA EN LA PERIFERIA
}

Archaeology in an environment of science in the periphery

\section{Roberto Valcárcel Rojas*}

Resumen: La arqueología que se practica en Cuba puede considerarse una disciplina científica marginal con relación al número de especialistas, producción científica, interacción académica y nivel de reconocimiento, entre otros aspectos. Si bien las determinantes propias de un país pobre son fundamentales para definir esta situación, el problema se vincula también con otros aspectos del ámbito científico, tecnológico y sociocultural. En este artículo se discuten tales problemáticas. Además se proponen líneas de acción que podrían contribuir a atenuar esta situación.

Palabras claves: Arqueología cubana, arqueología pública, comunidad científica.

* Doctor en arqueología por la Universidad de Leiden. Email: rvacarcel@holguin.inf.cu 
Abstract: The archeology practiced in Cuba can be considered as a marginal scientific discipline in terms of number of specialists, scientific production, academic interaction and recognition. The economic conditions of a poor country are important causes of this situation but the problem is also related with other aspects of the scientific, technological and sociocultural Cuban reality. This paper discuss this circumstances and proposes actions that could help to mitigate this problematic.

Keywords: Cuban archeology, public archeology, scientific community.

\section{Introducción}

Debido a su potencial económico y su nivel de desarrollo, Cuba forma parte de aquellos estados generadores de una actividad científica periférica, si se entiende como tal "la práctica científica que se efectúa en los países subdesarrollados y cuya expresión en términos de número de científicos, publicaciones, patentes y otros indicadores es realmente modesta a nivel mundial". (Núñez Jover, 2013: 113). Sin embargo, la valoración de sus avances científicos en determinados sectores, su capacidad de exportar servicios asociados a la ciencia, y el impacto que esta ha conseguido en muchas áreas de su sociedad, obligan a hablar más -al menos para ciertos espacios de la ciencia cubana- de ciencia en la periferia que de ciencia periférica.

Como en todos los países en desarrollo, la ciencia en Cuba sufre las dificultades propias de las economías pobres. A esto se suman problemáticas adicionales para el acceso a medios tecnológicos e información, así como para la interacción con los ambientes internacionales, generadas en gran medida por la situación de confrontación con los Estados Unidos, líder en numerosas esferas 
de la investigación científica a nivel mundial. Tales circunstancias determinan que se dé prioridad a ciertas áreas de la ciencia mientras que otras, aun cuando en términos de discurso e institucionalización consiguen el estatus general de valor atribuido a la ciencia, carecen del apoyo y la aceptación necesarios para su consolidación y crecimiento. Esto las relega a una posición simbólica y marginal que erosiona su papel social y afecta su credibilidad en la comunidad científica nacional e internacional.

La arqueología es una de estas disciplinas. Su práctica en Cuba es más antigua que la de algunas áreas de la ciencia hoy bien establecidas siendo muchos de sus actores, pasados y presentes, científicos e intelectuales notables. Pese a ello su estatus social e institucional puede considerarse marginal en términos de número de especialistas, producción científica, interacción académica y nivel de reconocimiento, entre otros aspectos. Si bien las determinantes propias de un país pobre son fundamentales en esta situación el problema se vincula también con otros aspectos de la realidad de carácter científico, tecnológico y sociocultural. Algunos de esos aspectos se discutirán en este artículo. Se considera pertinente proponer algunas líneas de acción que puedan contribuir a mitigar esta situación y valorar el impacto social que tiene el afianzamiento de la arqueología como ciencia.

\section{Ciencia en la periferia}

Núñez Jover (2013) ha caracterizado la labor científica en los países de la periferia desde los siguientes elementos:

- Las comunidades científicas se distinguen por la inferioridad numérica (casi nunca hay lo que se suele llamar "masas críticas", es decir, el número de personas que permitan articular un trabajo colectivo).

- Mayormente los procesos de legitimación de la ciencia tienen carácter exógeno (provienen de los países desarrollados), por lo 
que la agenda científica no tiene carácter nacional y se produce la institucionalización de relaciones de dependencia respecto a los países desarrollados en lo concerniente al proceso de formación, la actuación de los científicos, el progreso de la actividad de investigación así como de su financiamiento.

- La incorporación del científico de la periferia a la comunidad científica internacional suele ser parcial, con dificultades de integración real a los mecanismos y sistemas académicos, siendo con frecuencia muy alto su desplazamiento permanente hacia los espacios centrales.

- En la periferia la escasez de científicos, la indolencia social, la lentitud con que cristalizan las tradiciones de investigación, condicionan la fragilidad institucional de la ciencia.

- La ciencia, en muchos casos, es una ciencia académica (y a veces amateur) que carece de bases sociales y culturales, con bajo reconocimiento y retribución.

En lo que respecta a América Latina se distinguen (Anda e Iglesias, 1983) posiciones diversas entre las que pueden mencionarse:

(1) Las que estiman irrelevante el papel de la investigación científica e innecesaria su promoción;

(2) Las que ven la ciencia al servicio del desarrollo de la sociedad (desarrollistas) pero con una visión inmediatista, que puede conducir a perder de vista el papel de la investigación estratégica y termina en proyectos de transferencia desde los cuales se afirma la dependencia tecnológica, o a modelos de desarrollo beneficiosos para sectores minoritarios de la sociedad;

(3) La llamada posición cientificista, donde se plantea la producción de conocimientos solo con relación a la libertad académica y a la capacidad creativa del investigador, asumiendo la ciencia como autónoma, conducida por sus propios objetivos intrínsecos. 
Entendemos, como plantea Licha (1994), la necesidad de los países subdesarrollados de establecer indicadores propios para medir el impacto de la ciencia que se practica en ellos, como algo más circunspecto a sus necesidades y metas. La pertinencia de determinados indicadores para la evaluación de aspectos específicos del funcionamiento de la ciencia es un tema complejo por ello aquí solo esbozamos una reflexión generalizadora, sostenida desde los detalles más evidentes. Creemos que los criterios inicialmente citados, si bien definen la posición de la ciencia como sistema, pueden ser de utilidad para reconocer la ubicación de determinadas disciplinas en un ambiente de ciencia en país subdesarrollado. En este sentido y considerando los indicadores del caso cubano, los asumimos como guía para esbozar una evaluación del trabajo arqueológico en la Isla.

\section{Arqueología en Cuba}

La arqueología es la ciencia encaminada al estudio de las sociedades del pasado (también puede hacerlo con el presente) a través de sus restos materiales. En tanto pretende la comprensión del género humano y ya que se ocupa del pasado del hombre, es una ciencia social. El registro histórico hace declaraciones (las cuales deben interpretarse), sin embargo, los restos materiales no dicen de forma directa qué debemos pensar y requieren (como las ciencias técnicas y naturales) de procedimientos de interpretación donde se da la experimentación y creación de modelos (Renfrew y Bahn, 1998: 10). Su relación con la tecnología parte de las contribuciones ofrecidas por diversas especializaciones vinculadas a otras ciencias, como la geología, biología, química, física, etc., y con tecnologías derivadas de estas (dataciones radiocarbónicas, estudios de ADN antiguo, análisis cromatográficos para la identificación de residuos, estudios de prospección geofísica, prospección electromagnética, etc.). 
Desde sus inicios en Cuba, a fines del siglo XIX, la arqueología estuvo ligada a un grupo muy reducido pero prestigioso de intelectuales que enfatizaron en el estudio del pasado precolombino combinando técnicas de la antropología física con el estudio de las fuentes etnohistóricas hispanas y la lingüística. Dichos intelectuales se dedicaron a la colecta de restos materiales de estas sociedades. En las primeras décadas del siglo XX, la presencia de investigadores norteamericanos movió la labor arqueológica hacia una perspectiva normativa interesada en la identificación de culturas y sus rasgos materiales. Entre 1930 y 1959 se dio un período profesionalmente muy significativo, pues crece el número de cubanos involucrados en estos estudios que, usando como referencia inicial los aportes norteamericanos, adelantan varios intentos de generalización de datos arqueológicos a fin de explicar el pasado precolombino de la Isla.

En esta época se tiende a organizar el trabajo arqueológico tanto a nivel de normalización metodológica como institucional y legislativa. Se crean los grupos científicos Guama y Humboldt y la Comisión Nacional de Arqueología, institución capaz de vertebrar una publicación de rigor intelectual, conectar las labores cubanas con las organizaciones internacionales y reunir la cooperación de profesionales, aficionados y coleccionistas. Entre los últimos está la génesis de verdaderos museos locales y la extensión de las tareas investigativas a todo el país. Durante esos años, la arqueología logró un significativo reconocimiento gubernamental y su entrada a los Congresos Nacionales de Historia. El apoyo de prestigiosos intelectuales, el uso que se hace de sus informaciones y la permanente defensa del patrimonio nacional -enarbolada como tarea primordial- vitalizan su papel dentro de las ciencias sociales.

Con el triunfo de la Revolución cubana (1959) el proceso de profesionalización de la arqueología logra cristalizar en tanto la mayoría de los relacionados con la disciplina antes de este período lo hacían a partir de intereses personales y mayormente sin 
remuneración económica. La Revolución puso la cultura y la ciencia como objetivos prioritarios del nuevo proyecto social. Esto determinó un reordenamiento institucional facilitador del desarrollo del conocimiento en estas áreas y la creación de nuevos instrumentos legales al respecto. Una de las acciones más importantes fue la creación de la Academia de Ciencias de Cuba (ACC), como institución rectora del trabajo científico nacional y, como parte de esta, el Departamento de Antropología, fundado en 1962. Junto al Museo Montané, perteneciente a la Universidad de La Habana, y a la Sección de Arqueología de la Universidad de Oriente, en Santiago de Cuba, este Departamento se convertiría en motor del trabajo arqueológico en la Isla durante las décadas siguientes. Desde esta base se irían conformando instituciones y grupos de trabajo en distintas partes del país, que hoy se integran en el Ministerio de Ciencias, Tecnología y Medio Ambiente (CITMA), heredero de la antigua Academia de Ciencias de Cuba. Otras pertenecen al actual Ministerio de Cultura o al Ministerio de Educación Superior. Son estas las estructuras institucionales principales que desarrollan la labor de investigación arqueológica en la Cuba contemporánea.

Los años 70 y 80 fueron tiempos de consolidación y desde la ACC se organizaron proyectos de investigación de carácter nacional, procesos de formación de diverso nivel que incluyeron doctorados en países del antiguo campo socialista, y encuentros académicos de diverso tipo dirigidos a nuclear la discusión académica; puede decirse que existió algo muy cercano a una pequeña comunidad científica en arqueología. A partir de 1993 la crisis económica que afectó el país (conocida como periodo especial) generó daños importantes en el Sistema Nacional de Ciencia. En lo que respecta a la arqueología se produjo un éxodo masivo de personal y una dramática contracción de las investigaciones, especialmente de todo el trabajo de campo. En otros espacios institucionales, como el Ministerio de Cultura o de Educación, el proceso fue similar o 
más intenso. Los dos grupos de investigación principales de la antigua ACC, en La Habana y Holguín (Departamento Centro Oriental de Arqueología) respectivamente, para 1990 contaban con 22 investigadores y tres ayudantes de investigación, en el primer caso, y siete investigadores y dos ayudantes de investigación en el segundo caso. Para el año 2006, período en que producía cierta recuperación, La Habana contaba con 10 investigadores y tres ayudantes de investigación y Holguín con tres investigadores y cuatro técnicos de investigación. Para el 2013 el grupo de La Habana se mantenía con igual cantidad de investigadores y el de Holguín contaba con cuatro. Esto fue acompañado del retiro laboral de varios profesionales de larga experiencia, formados en universidades del antiguo campo socialista o con altos grados científicos, y de un deterioro masivo - no revertido- de la infraestructura de investigación y de museos del país.

En las últimas décadas ha existido un fuerte crecimiento de la arqueología histórica, particularmente tras la creación del Gabinete de Arqueología de la Oficina del Historiador de la ciudad de La Habana $(\mathrm{OHCH})$, institución centrada en apoyar los trabajos de estudio y restauración de La Habana Vieja. Se ha producido la aparición de algunos centros pequeños que intentan replicar este formato, con un impacto positivo en diversas áreas de la arqueología histórica y en los procesos de recuperación de ciertas urbes. Sin embargo, dada la estructura y tareas que se imponen a estas instituciones, adolecen de dificultades para lograr una adecuada proyección científica aun cuando es notoria la calidad del trabajo de muchos de sus especialistas y el serio impacto social que han logrado algunas.

La mayoría de los que practican arqueología en Cuba lo hacen desde una fuerte vocación profesional y con una enorme capacidad de consagración y esfuerzo personal, en condiciones de trabajo muy difíciles dada la falta de recursos, y con una remuneración y reconocimiento social muy limitados. No obstante, los aspectos 
propios de la ciencia periférica pueden reconocerse en el panorama ${ }^{1}$ antes descrito y resumirse en los siguientes elementos:

- La arqueología cubana, dada la escasez de personal e instituciones ha tenido dificultades para proyectarse como comunidad científica y para articular y sostener un trabajo colectivo. Esto se relaciona con el hecho de que no ha existido una carrera universitaria que garantice la formación ordenada y adecuada de personal. ${ }^{2}$ La formación posgraduada, oportunidad para reorientar hacia la arqueología a profesionales formados en disciplinas afines ha sido muy escasa. La preparación de doctores ha estado extremadamente limitada por la ausencia de espacios docentes, tribunales académicos doctorales y reconocimiento disciplinario, y por las notables dificultades internas y externas, tras la caída del campo socialista, para su formación internacional.

- Si bien la agenda científica responde a intereses nacionales, la misma debilidad en cuanto a institucionalización y formación ha contribuido a la búsqueda de espacios de legitimación foráneos, principalmente, en términos conceptuales. Se derivó hacia el pensamiento normativo promovido por expertos norteamericanos o hacia la visión marxista del campo socialista. Esto desestimuló, en alguna medida, la conformación de una posición más coherente con líneas propias del pensamiento cubano y también generó una situación de aislamiento,

1 Este panorama no es excepcional en la región y de hecho hasta pudiera ser el menos crítico si consideramos el caso de las restantes Antillas. José Oliver ha relacionado esto con prioridades económicas de países subdesarrollados, con la ausencia de posibilidades de empleo, con la incapacidad de los arqueólogos de conformar un grupo de opinión profesional capaz de comunicarse efectivamente con los políticos y con los que tienen el poder, y en alguna medida, con la falta de conexión de la sociedad con el pasado precolombino. (Valcárcel Rojas y Cooper 2011).

2 Recientemente en la Universidad de La Habana ha iniciado una carrera de estudio y manejo del patrimonio que incluye una especialización en arqueología, si bien con fuerte orientación hacia la arqueología colonial. 
posterior a 1959, respecto al pensamiento progresista latinoamericano o a la discusión científica de avanzada relacionada con centros académicos norteamericanos y europeos.

- Excepto en la ACC (actual CITMA), en el grupo de trabajo de la Universidad de La Habana, y de modo más reciente, en el Gabinete de Arqueología (OHCH), no se logró una institucionalización sistemática de la práctica arqueológica. Los grupos relacionados con centros de educación o espacios de cultura, con excepciones meritorias, han sido casi siempre pequeños e inestables y, generalmente, de baja productividad científica.

- Han existido grandes dificultades para lograr el nexo adecuado con la tecnología aplicada a la arqueología. Esto se ha conseguido en pocas instituciones, entre las cuales destaca la Universidad de La Habana, y no ha logrado consolidarse instucionalmente dicho sector de la investigación ya que carece de los recursos para acceder a estos servicios en espacios internacionales. Tal circunstancia influye en el alcance de los resultados de investigación o en la obtención de resultados científicos incompletos o deficientes.

- Pese a la calidad científica de muchos investigadores y al esfuerzo de algunas instituciones por mantener un trabajo serio y con impacto social, un número importante de los que se reconocen social e institucionalmente como arqueólogos desarrollan su trabajo desde una perspectiva amateur. Esto influye en el modo en que sectores consolidados de la ciencia valoran la arqueología, generándose una situación de descalificación del trabajo arqueológico también determinada por las dificultades para conseguir un discurso explicativo y una promoción sistemática y orgánica de los resultados de investigación, así como una integración seria al sistema de ciencias cubano.

- La práctica arqueológica de más calidad ha tendido a una posición cientificista, cerrada a un pequeño grupo de expertos 
o conectada a ciertas esferas de discusión internacional -también limitadas-, sin lograr una conexión sostenida y real entre resultados de investigación e intereses sociales. Sus publicaciones periódicas mayormente han sido escasas y de corta vida e igualmente la publicación de textos monográficos o de generalización, siendo mínima la producción de divulgación. Los espacios de discusión académica también han sido limitados e inestables concentrándose en la capital del país. Allí se han situado históricamente las instituciones más importantes y también la mayoría de los recursos creándose una jerarquización de la práctica arqueológica y de toda la labor de manejo del patrimonio arqueológico que muchas veces dificulta la concertación nacional entre instituciones e investigadores y el reconocimiento de los resultados de trabajo generados en el resto del país.

- La diversidad en los procesos y niveles de formación de los investigadores, proyección investigativa, inserciones institucionales y modo de trabajo, y el bajo nivel de concertación, intercambio y comunicación, cuestionan actualmente la existencia de una verdadera comunidad científica en la arqueología de Cuba, al menos en los términos planteados por Núñez Jover (1998: 81).

\section{Buscando causas}

Se cree que al arribo europeo, la mayor parte de la población indígena de Cuba estaba formada por comunidades agricultoras ceramistas poco jerarquizadas, pertenecientes al tronco etnolingüístico de origen aruaco. Aparentemente, opinión actualmente en debate, en el extremo occidental de la Isla existían reductos de grupos pescadores-cazadores-recolectores. La población de agricultores hacia 1492 se estima en unas 200, 000 personas. En el discurso histórico tradicional se enfatiza la idea de un reducido número de sobrevivientes al prohibirse su uso como trabajadores 
forzados (encomendados) en 1553 (Pérez de la Riva, 1972; Mira Caballos, 1997). Es irrefutable, no obstante, que la Isla conservaba una significativa cantidad de población a diferencia de territorios como Las Bahamas o Jamaica, donde esta había desaparecido o se había reducido al mínimo. Para 1556, según el gobernador Diego de Mazariegos, aún se reportaban unos 2,000 indios de los cuales 200 eran antiguos esclavos traídos de otros lugares (Marrero 1993: 353). Esta cifra recoge individuos residentes en las villas o en espacios cercanos, por tanto debió ser mucho mayor el número total dada la presencia de una amplia población de origen indígena fuera del control hispano, sobreviviendo aislada o que se había dispersado tras el colapso de la explotación minera y la disgregación de la encomienda.

La trágica historia de la destrucción de la sociedad indígena debido al impacto militar de la conquista hispana, las enfermedades, y a la intensidad de su explotación laboral, convirtió al indio (expresión colonial del indígena) en una referencia importante sobre la ilegitimidad del sistema colonial español y su naturaleza intrínsecamente injusta y cruel. Esta visión fue sostenida por diversos círculos intelectuales de la Isla y en alguna medida se convirtió en parte del fundamento histórico del independentismo cubano del siglo XIX. Desde esa época ha dominado la idea de que el indio no sobrevivió como grupo humano ni logró integrarse realmente a la sociedad colonial, si bien se reconoce, en fechas posteriores, la importancia de recuperar y estudiar su legado, formado por numerosos elementos lingüísticos y aspectos de la cultura material.

Desde sus inicios, la investigación arqueológica en Cuba se concentró en contextos relacionados con las culturas indígenas. Actualmente se considera que los agricultores aruacos habían arribado a Cuba en el siglo VI D. C. sin embargo, la presencia de los recolectores cazadores se inicia hace unos 5,000 años antes del presente, aunque algunos especialistas opinan que podría remontarse hasta unos 9,000 años antes del presente (Guarch Delmonte, 1990). Esta amplia presencia genera un importante patrimonio 
cultural y establece una ocupación humana que conforma el capítulo más extenso de la historia de la Isla.

Por decreto del Presiente de la República, en 1928, se designó una Comisión para que evitase la dispersión de la riqueza arqueológica de Cuba. En 1937, también por decreto Presidencial, se creó la Comisión Nacional de Arqueología. Sus objetivos eran la conservación y estudio de los monumentos y objetos precolombinos y coloniales, la formación del Mapa Arqueológico de Cuba y contribuir al desarrollo del Museo Arqueológico Nacional. La Comisión contaba con una sección de Arqueología Aborigen y otra de Arqueología Colonial. El reglamento de la Sección de Arqueología Aborigen regulaba las excavaciones y la investigación de sitios y objetos indígenas, así como su conservación, controlaba las colecciones privadas, la salida de objetos arqueológicos del país y establecía la declaración de monumentos nacionales.

En los años setenta se formuló un cuerpo de leyes (Patrimonio Cultural, 2002), aún vigentes, que ordenarán el manejo del patrimonio arqueológico considerado este como parte del patrimonio cultural de la nación cubana. La Ley N. ${ }^{\circ}$, "Ley de protección al patrimonio cultural" (1977), define los bienes que forman el patrimonio cultural de la nación, incluyendo en ellos los de carácter arqueológico. La Ley N. ${ }^{\circ}$ 2, "Ley de los Monumentos Nacionales y Locales" (1977) define y ordena el procedimiento para realizar excavaciones y exploraciones arqueológicas, disposiciones que se norman mediante el Decreto N. 55 , "Reglamento para la ejecución de la Ley de los Monumentos Nacionales y Locales" (1979). Este reglamento establece las categorías de Monumento Nacional y Monumento Local y su identificación, registro, protección y conservación. Define además las distintas tipologías que pueden considerarse en el rango de monumento nacional o local: Centros Históricos Urbanos, Construcciones, Sitios y Objetos. En estas dos últimas se consideran principalmente los elementos referidos al patrimonio arqueológico. 
En el caso de la arqueología el reglamento establece un fuerte control sobre los sitios arqueológicos, sobre las excavaciones y la posesión de objetos, prohibiendo las excavaciones no supervisadas científicamente y cualquier forma de afectación de los sitios y contextos, así como la salida de objetos del país. Crea una estructura organizativa que gira en torno a la Comisión Nacional de Monumentos - la cual posee una Subcomisión de Arqueología-, y las Comisiones Provinciales de Monumentos, encargadas de regular el manejo de los sitios y objetos.

En la última década se han definido nuevas regulaciones en torno a las actividades de investigación arqueológica y a la presentación de proyectos al respecto. Se ha establecido la Lista de profesionales de la Arqueología de Cuba, la cual recoge a los investigadores que se estiman, por la Subcomisión de Arqueología de la Comisión Nacional de Monumentos, capacitados para desarrollar excavaciones arqueológicas. Su objetivo es limitar las excavaciones realizadas por aficionados o personas que aún dentro de instituciones relacionadas con la protección del patrimonio carecen de la formación requerida para intervenir sitios arqueológicos.

Otro elemento clave en la estructura de estudio y control del patrimonio arqueológico es la Red de Museos de la República de Cuba. En los más de 160 municipios de Cuba hay al menos un museo, el cual ofrece una visión de la historia y la naturaleza de la región. Gran parte de estos museos municipales y de los mismos museos nacionales o provinciales, hay varios museos especializados en arqueología en Cuba, muestran material arqueológico y son el lugar desde el cual se trabaja con la población a fin de proteger el patrimonio en todas sus modalidades. Esto ha contribuido a popularizar el conocimiento de los objetos arqueológicos, ha limitado el surgimiento de nuevas colecciones privadas al divulgarse la legislación vigente y estimularse la donación de los objetos a los museos - siempre cercanos y abiertos a la comunidad-, y ha facilitado la rápida identificación de sitios arqueológicos. 
Sin dudas hay una conciencia nacional sobre la necesidad de proteger el patrimonio nacional y entre ellos, el arqueológico, cultivada en el trabajo de los museos con las comunidades y a través del proceso educativo a todos sus niveles; pese a ello la afectación al patrimonio sigue constituyendo un problema importante y con múltiples facetas (Robaina Jaramillo et al. 2003; Gutiérrez Calvahe et al. 2007). La Comisión Nacional de Monumentos es un órgano adscrito al Ministerio de Cultura que convoca para su funcionamiento, pero no de modo permanente, representantes de distintos ministerios e instituciones. Al ser un órgano integrador, que puede recabar el apoyo de cualquier institución o instancia del estado y la sociedad, queda implícita su capacidad para hacer valer las medidas de protección. Sin embargo, aunque la Comisión Nacional de Monumentos cuenta con grupos asesores para temas específicos, no tiene suficiente personal especializado en arqueología ni los recursos necesarios. Además pese a que tiene poder legal para prohibir la acción contra los monumentos, carece de instrumentos para hacer efectiva la prohibición dependiendo de los mecanismo legales externos. En este sentido aunque la Ley N. 62 (Código Penal, Título 6, Delitos contra el patrimonio culturah), establece sanciones de privación de libertad y multas por la realización de exploraciones y excavaciones ilegales, se hace muy difícil implementar su ejecución.

Con mucha frecuencia los procesos de inversión económica y de planificación de uso de los territorios y ciudades no consideran los elementos arqueológicos o los tratan de modo secundario. Hay representantes de instituciones relacionadas con dichos procesos que están integrados a la Comisión Nacional de Monumentos o a las comisiones provinciales, pero legalmente no existe un cuerpo de disposiciones que de modo preciso los involucre financieramente en el proceso de salvamento o estudio de estos. Por otra parte, su afectación casi nunca deriva en una respuesta o sanción que modifique las líneas de crecimiento o inversión o una recuperación real del patrimonio involucrado. En este contexto, el trabajo 
arqueológico, sin capacidad suficiente para enfrentar las necesidades de estudio y protección del patrimonio, no llega a reconocerse como una verdadera necesidad y obligación.

Esta situación supone una gran contradicción que nace en el modo social e institucional de entender nuestra historia y su valor directo en la defensa de la identidad nacional. No se reconoce un vínculo real entre el pasado precolonial de la isla y los procesos posteriores asociados a la dominación europea y posterior conformación de un ente nacional. En este ente tampoco se estima la presencia del indígena dada la ausencia de un segmento poblacional que pudiera reivindicar dicha base étnica. En tales circunstancias se ve como historia real y efectivamente conectada con nuestra identidad, la historia colonial y posterior, la cual se cree puede ser estudiada solo desde documentos históricos u otras fuentes y especialidades. En esta perspectiva el patrimonio cultural asociado al mundo indígena es un elemento cosificado que se recupera y protege para aludir al compromiso de la nación con la cultura pero sin un esfuerzo serio de comprensión e interpretación. Por tanto los arqueólogos, que científicamente pudieran asumir esta tarea, son solo necesarios para la acción puntual de captación y manejo inmediato de este patrimonio, por ello la arqueología se percibe como una herramienta limitada, cuyo fomenta solo se da para este fin. Al tener un carácter secundario en la visión nacional y ante las múltiples prioridades de sobrevivencia que enfrenta el país como nación subdesarrollada este patrimonio es pospuesto en términos de conservación y estudio. El patrimonio histórico sufre similar suerte cuando depende de procesos de manejo relacionados a la arqueología. Aquí aparece un efecto circular pues se prioriza lo que puede aportar la historia u otras disciplinas, como la arquitectura, a la comprensión y manejo de este patrimonio, acción en la que influye la subestimación con que en general se percibe a la arqueología, vista mayormente en su relación con la materialidad precolombina. De hecho, en muchos de los casos en que se integra la arqueología al estudio de espacios coloniales se 
le limita a una acción de recuperación y conservación, sin potenciar a fondo su capacidad real de explicar e interpretar el pasado y la sociedad. Esta postura retarda el desarrollo de la arqueología que no consigue convertirse en un interlocutor capaz de exigir el mejoramiento institucional o material de su estatus científico. Tal proceso necesariamente afecta su capacidad de llegar a la sociedad, estableciéndose un ciclo que conecta debilidad institucional, capacidad científica y reconocimiento social.

\section{Sobre soluciones}

A corto plazo el Estado cubano no podrá aportar los recursos que la arqueología requiere para su potenciación como ciencia, particularmente en las actuales condiciones de crisis económica global. De cualquier modo es importante que se entiendan los beneficios que tendría trabajar en esta dirección. La labor arqueológica tiene un impacto notable en la vida social y económica de muchos países, particularmente en aquellos que han hecho del pasado estudiado a través de la arqueología un símbolo de identidad capaz de dar coherencia y fortaleza a los estados y proyectos nacionales (Ulloa y Valcárcel Rojas, 2003; Veloz Maggiolo, 1999). Por otro lado, la labor arqueológica también se planifica para solucionar problemas concretos de enorme interés. Los trabajos que aportan datos sobre el manejo de los bosques y el ecosistema en sociedades antiguas, así como el empleo de sistemas agrícolas de bajo impacto medio-ambiental o de manejo del agua por estos pueblos, han sido muy útiles para enfrentar tareas del presente. Igual importancia han tenido las investigaciones sobre el uso de viejas fuentes nutritivas, sustancias medicinales y formas de curación, entre otras muchas antiquísimas soluciones humanas que hoy vuelven a ser útiles gracias a la arqueología.

Más allá de sus limitaciones hay ejemplos cubanos del valor social de la arqueología y de la posibilidad de hacer ciencia de calidad en el entorno de una disciplina periférica y marginada. El caso de 
la investigación del sitio arqueológico El Chorro de Maíta es uno de ellos y en él aparecen claves que pueden servir para favorecer el desarrollo de la disciplina a escala del país. Esta locación se ubica en el nororiente de Cuba, en la actual provincia de Holguín; el lugar conserva los restos de un amplio asentamiento indígena vigente a la llegada de los europeos. En 1941 el arqueólogo norteamericano Irving Rouse (1942: 103-106) lo explora y presenta un reporte de su visita y de los materiales arqueológicos del lugar. Entre 1986 y 1988, los arqueólogos del Departamento de Arqueología de Holguín, bajo la dirección del Dr. José Manuel Guarch Delmonte, localizaron y excavaron un cementerio (Guarch Delmonte 1994, 1996), el único reportado hasta ahora en este tipo de comunidades en la Isla. (Valcárcel Rojas, 2008).

Entre 1989 y 1990, una vez retirados todos los entierros del espacio excavado de manera controlada, sobre el área del cementerio se construyó un museo. Este muestra reproducciones de los restos humanos y objetos obtenidos en el sitio. Las evidencias originales se trasladaron al Departamento Centro Oriental de Arqueología para su estudio. Cerca del museo se levantó una recreación de una aldea indígena que ilustra, usando figuras humanas a escala natural, cómo pudo ser la vida de las personas que estaban enterradas en el cementerio, e incluso la posible estructura de la misma aldea que debió rodear al cementerio.

El lugar constituye un espacio de interés turístico pero también un recurso educativo de gran valor. Se vincula al cercano polo turístico de Guardalavaca y resulta una importante oferta extra hotelera que muestra detalles de la historia cubana a visitantes extranjeros y nacionales y obtiene ingresos por diversas vías. La construcción del museo y la aldea supuso mejoras de infraestructura para la comunidad. Se construyó una carretera y se logró acceso a distintos servicios que antes no llegaban a esta zona alejada. También se abrieron fuentes de empleos y posibilidades de superación profesional para muchos de los vecinos, algunos de los cuales hoy trabajan en el mismo museo o para el turismo. 
Sobre el sitio hay todo un poblado rural con más de 400 habitantes, así que la excavación del cementerio, ubicado bajo varias casas, permitió salvar materiales arqueológicos de enorme importancia. Por otro lado, la existencia del museo garantiza la vigilancia y protección del resto del lugar y una permanente labor de trabajo comunitario con un impacto muy positivo en la vida del asentamiento y en el fomento de nuevas actitudes y valores entre los que se encuentra el interés por el pasado y la historia local.

El museo también acoge a los investigadores que estudian el sitio. Desde el año 2005 se han venido sucediendo proyectos de investigación del Departamento Centro Oriental de Arqueología en Holguín, con apoyo de instituciones como la Universidad de Leiden, la Universidad de Alabama, University College London, Universidad de Pensilvania, entre otras, que han permitido continuar las excavaciones y los estudios. Las investigaciones de estas colecciones y del sitio sirvieron de base a varias disertaciones doctorales y de maestría, de investigadores cubanos, holandeses y de los Estados Unidos. Los resultados del trabajo científico son insertados en el museo a través de su guión expositivo y de la información que se ofrece a los visitantes. Es también el museo la institución reguladora de la participación de la comunidad en los trabajos arqueológicos en ejecución y quien transmite a esta lo nuevo que se conoce a partir de los estudios científicos.

La colaboración internacional permitió el acceso a recursos, informaciones y análisis diversos, no disponibles en Cuba, como fechados radiocarbónicos, estudios de arqueometalurgia, de determinación de residuos en vasijas de cerámica, de identificación de textil, coral y ámbar, datos documentales del Archivo General de Indias (España), entre otros. Gracias al apoyo de la Universidad de Alabama se pudieron reactivar, tras 20 años de interrupción, los trabajos de campo (excavaciones y prospecciones), realizándose además talleres de capacitación en temas de investigación cerámica y arqueología ambiental. Los aspectos biológicos fueron investigados a partir de la cooperación con el Grupo de Estudios del 
Caribe de la Universidad de Leiden, e incluyeron estudios de antropología física, tafonomía y análisis de origen territorial. Se colaboró en otras áreas de trabajo con instituciones cubanas, como los gabinetes de Arqueología y de Arqueometría de la OHCH. Este esquema de trabajo permitió estructurar una labor científica de alta calidad y con reconocimiento académico en ámbitos nacionales y extranjeros.

Las investigaciones más recientes en El Chorro de Maíta no solo destacan por una complejidad tecnológica que las pone a nivel de los estándares internacionales, sino por el impacto de sus resultados. Estos cambian de modo radical la visión del sitio y establecen la enorme importancia de las situaciones verificadas en el lugar a partir de la interacción indígena con los europeos (Valcárcel Rojas, 2012). Se determina que en la primera mitad del siglo XVI gran parte de las zonas no funerarias pudieron estar funcionando en circunstancias de control hispano del asentamiento, un pueblo de indios encomendados, y que el cementerio es el resultado principalmente de la mortalidad generada durante este período. La concepción del cementerio no es indígena, aun cuando no alcance la formalización de un cementerio cristiano típico. Este alberga restos de individuos indígenas cubanos y no cubanos, y al menos un africano. Es una composición etnodemográfica hasta ahora no identificada con este nivel de precisión en sitios indígenas del Caribe. Se explica como resultado de la inhumación de población encomendada y esclavos de diverso origen, radicados en el lugar o en espacios cercanos, en tanto hay indicios de un fuerte manejo colonial de la región. Tal dato, pionero en términos arqueológicos, se completa con la identificación de individuos posiblemente mestizos, valorándose detalles de una identidad y existencia que los sitúa, junto a indios y negros, en los escalones inferiores de la estructura social del momento. Se hace visible la acción de cristianización de ciertos individuos, parte de los grupos de élite indígena, y de construcción de sujetos coloniales como el "indio". De modo paralelo se distingue el sostenimiento de prácticas 
tradicionales, evidencia de una posición activa del indio, expresada además en soluciones sincréticas. En este entorno múltiple, verdadero escenario transcultural, aparecen nuevas identidades e individuos; en ellos confluye una mezcla genética y cultural la cual será marca de los nuevos tiempos y de la futura conformación del ente cubano.

Se trata de un acercamiento valioso a la vida y la muerte en espacios coloniales, fuera de las villas hispanas, donde la arqueología es un recurso verdadero para potenciar la visión de esos "otros" individuos y ambientes. Este caso es muy relevante pues se analiza un espacio indígena, un pueblo encomendado, transformado en un complejo escenario de dominación y supervivencia, pero sobre todo porque supone la continuidad y persistencia de este componente en momentos donde la historia tradicional solo ve su final. Es un aporte útil en la revisión de la historia cubana y caribeña a fin de construir una imagen menos colonial de nuestra región, una historia donde se reconozca la real participación del indígena y la complejidad de los procesos donde se vio insertado.

El caso de El Chorro de Maíta tiene que ver mucho con el reconocimiento que la arqueología tiene en la provincia de Holguín, tras años de trabajo y de influir en la conciencia y la cultura local. Esto determinó que los niveles políticos y de gobierno apoyaran la construcción de un museo que sigue siendo único en Cuba porque en otras partes de la Isla, aun cuando existe un patrimonio arqueológico valioso, se tiene una visión diferente de la importancia de la arqueología. Tal circunstancia ha sido determinante en la sobrevivencia institucional de la arqueología en Holguín y en la percepción y manejo del patrimonio indígena como un símbolo de identidad regional. Lo dicho demuestra que la investigación arqueológica en Cuba puede generar impactos de provecho económico y social, detalle que en el caso holguinero fue replicado en lugares como el parque Nacional Bariay y diversos espacios asociados al turismo. Prueba la necesidad de ampliar la colaboración entre las instituciones cubanas para aprovechar mejor los recursos, 
conocimientos y experiencias disponibles, y que la colaboración internacional, desde las normas de respeto y cooperación académica, no solo es posible sino necesaria.

\section{Conclusiones}

En las actuales circunstancias, el futuro de Cuba y de su modelo social se entienden enormemente ligados al tema del desarrollo económico; no existen posibilidades de que la arqueología alcance un papel importante en el esquema de ciencias cubano si no logra una práctica más seria y profesional y, por tanto, menos periférica y con mayor impacto social. Desarrollo social es mucho más que crecimiento económico (Núñez Jover, 2002) y sería absurdo crecer perdiendo lo conseguido en términos de cultura y valores sociales (entre ellos los patrimoniales) o sin hacer de estos parte del concepto de crecimiento. Las soluciones por el momento, reconociendo lo discutido y el éxito de algunos proyectos, como el desarrollado en El Chorro de Maíta, pudieran moverse en estas direcciones:

- Lograr espacios de reconocimiento local y esquemas de crecimiento descentralizados, que permitan la sostenibilidad institucional de la disciplina apelando a recursos e intereses territoriales.

- Profundizar en los mecanismos de divulgación científica y en el manejo de los medios de difusión masiva, que en el caso cubano, generalmente, son receptivos a los resultados de esta disciplina, para conseguir respaldo y reconocimiento social.

- Posición de autoanálisis que enfatice en priorizar la calidad del trabajo científico, el fortalecimiento de valores éticos y la conformación de una verdadera comunidad científica nacional en arqueología, capaz de defender la importancia de la disciplina y los intereses de sus miembros. 
- Creación de nuevos espacios de diálogo científico en arqueología, que rompan con la tendencia a privilegiar a la capital del país como centro rector de la disciplina y potencien la diversidad en las circunstancias de encuentro y el reconocimiento de los distintos valores y trayectorias locales y regionales.

- Incremento de la colaboración entre instituciones nacionales y en el flujo de información científica y posibilidades de apoyo mutuo.

- Aumentar el impacto de la arqueología en los espacios educativos y en las esferas de manejo y estudio ambiental y social, a fin de generar interés en la disciplina y nuevas posibilidades de empleo e institucionalización del personal.

- Abogar permanentemente por la creación de una carrera de arqueología.

- Desarrollo de cursos de posgrado a nivel nacional e internacional que aporten la especialización requerida y potencien las bases académicas que el país fomenta en áreas afines a la arqueología (historia, sociología, biología, geografía, etc.)

- Trabajo en investigaciones que consigan la excelencia requerida y el reconocimiento académico nacional e internacional, pero que incluyan mecanismos para lograr un impacto social concreto considerando variables como el trabajo comunitario y el manejo y la promoción del patrimonio.

- Búsqueda del intercambio con otras disciplinas científicas a fin de poder hacer un uso más eficiente de los recursos científicos y tecnológicos del país en la investigación arqueológica e incrementar el reconocimiento del trabajo arqueológico y su calidad.

- Insistir en la necesidad de resultados que tengan un impacto real en la descolonización de la historia cubana al demostrar la continuidad e importancia de los procesos socioculturales previos al arribo europeo. 
- Insistir en la conexión del patrimonio arqueológico con la identidad nacional y en la necesidad de su defensa y conocimiento.

- Incremento de la colaboración internacional, desde normas de respeto y verdadera cooperación académica.

\section{Referencias bibliográficas}

Anda, E., \& Iglesias, R. (1983). La actividad científica en los países dependientes. Revista Mexicana de Física 1(30), 119-137.

Cuba. Dirección Nacional de Patrimonio Cultural. (2002). Protección del Patrimonio Cultural. Compilación de textos legislativos. La Habana: Consejo Nacional de Patrimonio Cultural y el Ministerio de Cultura.

Guarch Delmonte, J. M. (1990). Estructura para las comunidades aborigenes de Cuba. Holguín, Cuba: Ediciones Holguín.

Guarch Delmonte, J. M. (1994): Yaguajay, Yucayeque, Turey. Holguín: Publicigraf-Ediciones Holguín.

Guarch Delmonte, J. M. (1996). La muerte en Las Antillas: Cuba. El Caribe Arqueológico 1, 12 -25.

Gutiérrez Calvahe, D., Fernández Ortega, R. \& González Tendero, J. B. (2007). La conservación del patrimonio rupestrológico cubano. Situación actual y perspectivas. Gabinete de Arqueología 6,107-124.

Licha, I. (1994). Indicadores endógenos de desarrollo científico y tecnológico y de gestión de la investigación. En E. Martínez (Ed.). Ciencia, tecnologia y desarrollo: interrelaciones teóricas y metodológicas. (pp. 349-386). Caracas: Editorial Nueva Sociedad.

Marrero, L. (1993). Cuba: Economía y sociedad. Antecedentes. Siglo XVI (la presencia europea). (T. 1). Santo Domingo: Editorial Playor. 
Mira Caballos, E. (1997). El indio antillano: Repartimiento, encomienda y esclavitud (1492-1542). Sevilla: Muñoz Moya.

Núñez Jover, J. (1998). Material docente del curso Epistemología y educación. La Habana: Universidad de La Habana.

Núñez Jover, J. (2002). Ética, ciencia y tecnología: Sobre la función social de la tecnociencia. ILUIL, 25, 459-484.

Núñez Jover, J. (2013). La ciencia y la tecnología como procesos sociales. Lo que la educación científica no debería olvidar. Recuperado de http://www.campusoeiorg/salactsi/nunez00.htm

Pérez de la Riva, J. (1972). Desaparición de la población indígena cubana. Revista de la Universidad de La Habana, 196-197, 68-83.

Renfrew, C., \& Bahn, P. (1998). Arqueología: Teorías, métodos y práctica. Madrid: Ediciones Akal.

Robaina Jaramillo, J. R., Celaya, M., \& Pereira, O. (2003). La arqueología en la construcción de un discurso sobre identidad cultural en Cuba. Catauro. Revista cubana de antropología, 8, 47-61.

Rouse, I. (1942). Archaeology of the Maniabón Hills, Cuba. New Haven: Yale University Press.

Ulloa, J., \& Valcárcel Rojas, R. (2003). Reflexiones sobre la arqueología como historia. Boletín del Museo del Hombre Dominicano, 34, 73-82.

Valcárcel Rojas, R. (2008). Las sociedades agricultoras ceramistas en Cuba. Una mirada desde los datos arqueológicos y etnohistóricos. El Caribe Arqueológico, 11, 2-19.

Valcárcel Rojas, R. (2012). Interacción colonial en un pueblo de indios encomendados. El Chorro de Maita, Cuba. (Tesis doctoral). Leiden: Leiden University. Recuperado de https://openaccess.leide nuniv.nl/ handle/1887/20153. 
Valcárcel Rojas, R., \& Cooper, J. (2011). Temas de arqueología caribeña. El Caribe Arqueológico, 12, 99-109.

Veloz Maggiolo, M. (1999). Arqueología, historia e identidad. El Caribe Arqueológico 3, 20-27.

\section{Roberto Valcárcel Rojas}

Roberto Valcárcel Rojas (Holguín, Cuba 1968). Doctor en arqueología por la Universidad de Leiden; miembro del grupo de investigaciones del Caribe de la Universidad de Leiden e investigador auxiliar en el Departamento Centro Oriental de Arqueología, Ministerio de Ciencias, Holguín. Actualmente dirige el proyecto de investigación Estudios de cultura material en espacios de interacción indo-hispana. Autor de libros y numerosos artículos sobre arqueología precolonial y colonial de Cuba y el Caribe. Se especializa en temas de organización social indígena e interacción colonial temprana. Es uno de los coordinadores de la revista El Caribe Arqueológico.

Email: rvacarcel@holguin.inf.cu

Recibido: 15/10/2013 Aprobado: 08/02/2014 